\title{
面向电化学储能与转化的表界面工程
}

于乐 ${ }^{1, *}$, 黄小青 ${ }^{2}$, 张桥保 ${ }^{3}$, 张志成 4

1 北京化工大学, 有机无机复合国家重点实验室, 化学工程学院, 北京 100029

2 厦门大学, 固体表面物理化学国家重点实验室, 化学化工学院, 福建 厦门 361005

3 厦门大学, 材料学院, 福建厦门 361005

4 天津大学, 天津市分子光电科学重点实验室, 理学院化学系, 天津 300072

\section{Surface and Interface Engineering for Electrochemical Energy Storage and Conversion}

\author{
Le Yu ${ }^{1, *}$, Xiaoqing Huang ${ }^{2}$, Qiaobao Zhang ${ }^{3}$, Zhicheng Zhang ${ }^{4}$ \\ ${ }^{1}$ State Key Lab of Organic-Inorganic Composites, College of Chemical Engineering, Beijing University of Chemical Technology, \\ Beijing 100029, China. \\ ${ }^{2}$ State Key Laboratory of Physical Chemistry of Solid Surfaces, College of Chemistry and Chemical Engineering, Xiamen University, \\ Xiamen, 361005, Fujian Province, China. \\ ${ }^{3}$ College of Materials, Xiamen University, Xiamen 361005, Fujian Province, China. \\ ${ }^{4}$ Tianjin Key Laboratory of Molecular Optoelectronic Sciences, Department of Chemistry, School of Science, Tianjin University, \\ Tianjin 300072, China.
}

Email: yule@mail.buct.edu.cn

Published online: September 13, 2021.

随着不可再生化石燃料的枯竭和人类能源需 求的快速增长, 能源利用正从传统化石能源主体 逐渐转向低碳可再生能源。2020年9月, 习近平总 书记在第75届联合国大会上提出: “中国将在未来 几十年里, 采取一系列方法、政策和措施, $\mathrm{CO}_{2}$ 排 放力争于 2030 年前达到峰值, 争取在 2060 年前实 现碳中和。” 当前, “碳达峰、碳中和” 显然已成为 社会各界热议的焦点, 高效的能源转换和储存已 被视为最重要的全球挑战之一。

电化学能源转换技术可以完成电能和化学能 间的互相转化, 是实现清洁能源存储、转运及应用 的一项关键技术, 如电池、电容与电催化等。其中, 电催化更是电化学能源转化和存储、绿色合成, 环 境和电化学工程的核心基础, 其反应都发生在催 化剂表面, 涉及反应物, 反应中间体与催化剂表面 的相互作用, 同时电催化反应也涉及到电子、离子 和分子的转移 1 。一方面, 活性位点大多在催化剂 表面, 尺寸、结构、形貌、缺陷等状态将强烈影响 催化剂的催化活性; 另一方面, 反应三相界面间的 质量和电荷传递速率也关系着催化剂活性高低。
电极材料的表面和界面可以暴露活性位点的数 目、电荷转移的效率、中间体的吸附能力的强弱, 均成为电催化性能调控的重要切入点。此外, 对于 电池和超级电容器的性能研究也均与电极表面、 电极-电解液界面和催化剂表面的物化性质息息 相关。

由于表界面电化学行为涉及界面处化学/电化 学反应、结构/组分影响、热力学/动力学等相关行 为, 使得深入理解表界面化学反应的内在机理以 及限制因素, 并设计合理的表面功能化方法, 有助 于实现从本质上对电极材料性能的调控和提升, 对优化电极整体电化学性质有着重要的指导意 义。对此许多科研人员已研发出了一系列的表面 功能化方法, 如表面缺陷调控、轨道调控、元素掺 杂、异质结设计、表面保护、以及界面工程等, 并 系统研究了其在电催化材料及储能材料等中的关 键作用。

基于此, 本特刊专辑邀请了国内部分从事电 化学储能与转化的学者团队, 介绍他们近年来在 面向电化学储能及转化的表界面工程方面的重要 
研究进展和综述。下面对这些研究和总结进行扼 要介绍。

氢气是一种清洁高效的能源载体, 通过海水 电解规模化制备氢气能够为应对全球能源挑战提 供新的机遇。邓意达和胡文㮏等 ${ }^{2}$ 重点总结了具有 高选择性和强耐腐蚀性电极材料的设计合成与作 用机制, 详细介绍了如多金属氧化物、 $\mathrm{Ni} / \mathrm{Fe} / \mathrm{Co}$ 基 复合材料、氧化锰包覆异质结构等对氧气生成选 择性的研究进展及各种材料的抗腐蚀工程研究成 果。

微生物在自然界中广泛存在, 具有结构、组成 和代谢丰富的特点, 可作为电催化剂的模板以及 碳、磷、硫等非金属元素以及金属元素的来源, 已 成为电催化剂制备的新趋势。刘宏和王海青等 3 综 述了微生物 “智能”引导制备电催化剂的发展及在 电催化析氢(HER)、电催化析氧( OER)、氧还原反 应 $(\mathrm{ORR})$ 、二氧化碳还原 $\left(\mathrm{CO}_{2} \mathrm{RR}\right)$ 、锂电池 $(\mathrm{LBs})$ 等 领域的应用现状。

邵明飞和谢文富等 ${ }^{4}$ 从电极角度出发对电催 化二氧化碳还原反应 $\left(\mathrm{E}-\mathrm{CO}_{2} \mathrm{RR}\right)$ 领域中一体化电 极(包括一体化金属基、一体化金属化合物基和一 体化金属单原子基)的应用进行了综述。详细介绍 了不同一体化电极的设计思路, 以及结构和表界 面调控对 E- $\mathrm{CO}_{2} \mathrm{RR}$ 性能的影响规律, 重点探讨了 一体化电极对 $\mathrm{E}-\mathrm{CO}_{2} \mathrm{RR}$ 的构效关系及性能提升机 制等。

氧化亚硅 $(\mathrm{SiO})$ 作为锂离子电池负极材料, 具 有较高的理论比容量 $\left(2043 \mathrm{mAh} \cdot \mathrm{g}^{-1}\right)$ 以及合适的 脱锂电位 $(<0.5 \mathrm{~V})$, 且原料储量丰富、制备成本较 低、对环境友好, 被认为是下一代高能量密度锂离 子电池负极极具潜力的候选材料之一。张桥保和 张力等5综述了近几年来 $\mathrm{SiO}$ 负极结构和界面优化 的研究工作, 并从纯 $\mathrm{SiO}$ 的结构优化、 $\mathrm{SiO} / \mathrm{C}$ 复合和 $\mathrm{SiO} /$ 金属复合等三方面进行详细阐述, 并分别就 其方法特征、电化学性能及存在问题等进行了总 结与讨论。

席聘贤等 ${ }^{6}$ 提出使用有机配体用以表面改性 $\mathrm{NiCo}_{2} \mathrm{O}_{4}$ 纳米线, 并通过 $x$ 射线光电子能谱分析表 明, $\mathrm{Co}^{2+} / \mathrm{Co}^{3+}$ 比值的增加是其在碱性介质中用作 水分裂双功能催化剂电化学性能优异的主要原 因, 进而使得整体效率明显提高。

李伟等7采用了一种 “软硬双模板” 的策略合 成了兼具大孔和介孔的三维碳-碳化钛复合材料。 通过多孔结构的合理设计和高导电性 $\mathrm{TiC}$ 纳米颗 粒的引入, 缓解金属锂充放电过程中的体积膨胀 和调控金属锂的成核行为, 实现了无枝晶锂金属
负极的构筑。

木土春等 8 以花状 $\mathrm{NH}_{2}-\mathrm{MIL}-125(\mathrm{Ti})$ 为前驱体 和硬模板, 合成出具有花状结构的超细纳米 $\mathrm{TiO}_{2} /$ 多孔氮掺杂碳片复合物(记为FL- $\mathrm{TiO}_{2} / \mathrm{NPC}$ )。一方 面, 二维褶皱纳米片包含 $\mathrm{TiO}_{2}$ 纳米颗粒可以增大 活性物质与电解液的接触面积; 另一方面, 氮掺杂 多孔碳基体可以提高整体复合物的导电性和结构 完整性, 并表现出优异的电化学性能。

液流电池因为具有高储能效率, 低成本, 以及 可解耦的能源储存和功率输出设计, 被广泛认为 是适用于大型储能的首选技术。从广涛和卢怡君 ${ }^{9}$ 综述了液流电池的工作机制, 以提升非水系有机 液流电池的储能密度的策略为重点, 总结了非水 系液流电池中有机活性材料的研究进展, 讨论了 这些策略存在的问题和未来的发展方向。

吴锋, 赵腾及陈人杰等 ${ }^{10}$ 利用化学氧化法以三 苯胺(TPA)为原料成功合成了廉价的电压敏感聚 合物聚三苯胺(PTPAn), 并制备出电压敏感性涂层 隔膜(PTPAn/PP)。研究结果表明, 当PTPAn浓度为 $10 \%$ 时, 该功能隔膜(YP-2)具有最优的综合性能, 且该隔膜具有优异的多孔结构及连续的导电结 构, 与电解液具有良好的亲和能力, 可有效抑制锂 硫电池的过充并对锂硫电池进行充分的保护而不 影响其性能。

刘继磊及高鹏等 ${ }^{11}$ 从离子传输、枝晶形核与生 长、及安全性能三个方面详细探讨了隔膜对电池 性能影响的关键因素及其改性方法, 并系统总结 了隔膜结构、物化特性、力学性能、热学性能以及 电化学性能的表征技术, 以期为功能隔膜的合理 设计, 从而优化锂离子电池性能提供理论和实践 指导。

希望《物理化学学报》的广大读者能够喜欢这 些文章, 阅读愉快, 并从中有所启迪。

\section{References}

(1) Wang, C. M.; Bai, S.; Xiong, Y. J. Chin. J. Catal. 2015, 36, 1476. doi: 10.1016/S1872-2067(15)60911-1

(2) Cui, B. H.; Shi, Y.; Li, G.; Chen, Y. N.; Chen, W.; Deng, Y. D.; Hu, W. B. Acta Phys. -Chim. Sin. 2022, 38, 2106010. [崔柏桦, 施毅, 李 根, 陈亚楠, 陈伟, 邓意达, 胡文涁. 物理化学学报, 2022, 38, 2106010.] doi: 10.3866/PKU.WHXB202106010

(3) Ma, M. J.; Feng, Z. C.; Zhang, X. W.; Sun, C. Y.; Wang, H. Q.; Zhou, W. J.; Liu, H. Acta Phys. -Chim. Sin. 2022, 38, 2106003. [马明军, 冯 志超, 张小委, 孙超越, 王海青, 周伟家, 刘宏. 物理化学学报, 2022, 38, 2106003.] doi: 10.3866/PKU.WHXB202106003 (4) Song, Y. K.; Xie, W. F.; Shao, M. F. Acta Phys. -Chim. Sin. 2022, 38, 
2101028. [宋雨珂, 谢文富, 邵明飞. 物理化学学报, 2022, 38, 2101028.] doi: 10.3866/PKU.WHXB202101028

(5) Zhu, S. Y.; Li, H. Y.; Hu, Z. L.; Zhang, Q. B.; Zhao, J. B.; Zhang, L. Acta Phys. -Chim. Sin. 2022, 38, 2103052. [朱思颖, 李辉阳, 胡忠 利, 张桥保, 赵金保, 张力. 物理化学学报, 2022, 38, 2103052.] doi: 10.3866/PKU.WHXB202103052

(6) Sun, K.; Zhao, Y. Q.; Yin, J.; Jin, J.; Liu, H. W.; Xi, P. X. Acta Phys. -Chim. Sin. 2022, 38, 2107005. [孙辑, 赵永青, 殷杰, 靳晶, 刘翰文, 席聘 贤. 物理化学学报, 2022, 38, 2107005.] doi: 10.3866/PKU.WHXB202107005

(7) Zhang, W.; Liang, H. C.; Zhu, K. R.; Tian, Y.; Liu, Y.; Chen, J. Y.; Li, W. Acta Phys. -Chim. Sin. 2022, 38, 2105024. [张威, 梁海琛, 朱科 润, 田泳, 刘瑶, 陈佳音, 李伟. 物理化学学报, 2022, 38, 2105024.] doi: 10.3866/PKU.WHXB202105024

(8) Yang, Y.; Zhu, J. W.; Wang, P. Y.; Liu, H. M.; Zeng, W. H.; Chen, L.;
Chen, Z. X.; Mu, S. C. Acta Phys. -Chim. Sin. 2022, 38, 2106002. [杨 越, 朱加伟, 王鹏彦, 刘海咪, 曾炜豪, 陈否, 陈志祥, 木士春. 物 理化学学报, 2022, 38, 2106002.] doi: 10.3866/PKU.WHXB202106002

(9) Cong, G. T.; Lu, Y. J. Acta Phys. -Chim. Sin. 2022, 38, 2106008. [从 广涛, 卢怡君. 物理化学学报, 2022, 38, 2106008.] doi: 10.3866/PKU.WHXB202106008

(10) Zhang, N. X.; Wei, Z. Z.; Feng, T.; Wu, F.; Zhao, T.; Chen, R. J. Acta Phys. -Chim. Sin. 2022, 38, 2107009. [张楠祥, 魏壮壮, 冯涛, 吴锋, 赵腾, 陈人杰. 物理化学学报, 2022, 38, 2107009.] doi: 10.3866/PKU.WHXB202107009

(11) Mo, Y.; Xiao, K. K.; Wu, J. F.; Liu, H.; Hu, A. P.; Gao, P.; Liu, J. L. Acta Phys. -Chim. Sin. 2022, 38, 2107030. [莫英, 肖迕達, 吴剑芳, 刘辉, 胡爱平, 高鹏, 刘继否. 物理化学学报, 2022, 38, 2107030.] doi: 10.3866/PKU.WHXB202107030 